\title{
COMPARAÇÃO ENTRE INJEÇÃO NA COLUNA (“ON-COLUMN") E HEADSPACE DINÂMICO NA DETERMINAÇÃO DE BENZENO, TOLUENO E XILENOS (BTX) EM AMOSTRAS DE ÁGUA
}

Elaine A. A. F. Gobato e Fernando M. Lanças*

Universidade de São Paulo, Instituto de Química de São Carlos, CP 780, 13560-970 São Carlos - SP

Recebido em 18/10/99; aceito em 16/11/00

\begin{abstract}
COMPARISON BETWEEN ON-COLUMN INJECTION AND DYNAMIC HEADSPACE IN THE DETERMINATION OF BENZENE, TOLUENE AND XYLENE (BTX) IN WATER. The analysis of water samples containing volatile organic compounds has become an important task in analytical chemistry. Gas chromatography has been widely used for the analysis of volatile organic compounds in water. The headspace analysis shows as a principal characteristic the possibility of determination of the volatile components in drinking water. Benzene, Toluene and Xylene (BTX) are important compounds usually present in drinking water, from contamination by petroleum derivatives. Since they are toxic compounds even when present in low concentration levels, their determination is important in order to define the quality of the water. The sampling technique using headspace, coupled with gas chromatography as the separation method, showed to be suitable for BTX analysis in several samples at the $\mu \mathrm{g} / \mathrm{L}(\mathrm{ppb})$ level.
\end{abstract}

Keywords: water analysis; dynamic headspace; petroleum derivatives.

\section{INTRODUÇÃO}

Com o crescente aumento da população e da atividade industrial, o tratamento e condicionamento da água potável, assim como a proteção do meio ambiente, tem se tornado uma das principais preocupações da sociedade moderna. A manutenção de condições mínimas de qualidade de água deve ser uma meta de principal importância para a população ${ }^{1}$. Um fornecimento adequado de água potável limpa é uma das necessidades primárias para uma boa saúde ${ }^{2}$.

A garantia da qualidade, baseada em sistemas de tratamento bem projetados e em análises laboratoriais de controle, é a esperança de milhões de pessoas. É preciso preservar os mananciais, custe o que custar ${ }^{1}$, pois muitos compostos orgânicos encontrados em água de rio e na água potável tem atividade carcinogênica ${ }^{3}$.

Essa contaminação de rios por compostos orgânicos voláteis ("VOC", COV) pode ocorrer por descarga direta ou por lixiviação de lixo químico depositado ao longo do rio ${ }^{4}$, tornando a análise de traços de compostos orgânicos em água, um dos maiores problemas enfrentados pela química analítica moderna.

Dentre os métodos modernos de análise química, a cromatografia ocupa, sem dúvida, um lugar de merecido destaque no que se refere à separação, identificação e quantificação de espécies químicas 5 .

Quando a concentração das amostras de interesse é muito baixa, inferior ao limite de detecção do detector, o enriquecimento da amostra torna-se necessário. Várias técnicas de enriquecimento baseadas em princípios físico-químicos estão disponíveis, entre elas, a extração líquido-líquido ("LLE”, ELL), destilação, análise por headspace e extração por fase sólida (“SPE”, EFS).

O headspace é uma técnica excelente e sensível, utilizada para analisar compostos em baixas concentrações. Nesta técnica, na qual o analito é, necessariamente, mais volátil que a matriz, este volatiliza preferencialmente, podendo ser determinado sem os interferentes dos outros componentes da amostra, através da análise do vapor desprendido do analito ${ }^{6}$.

A principal característica do headspace é a possibilidade da determinação de componentes voláteis da amostra a ser estudada de forma direta. Além disso, o headspace torna-se insubstituível e muito eficiente, pois possibilita a introdução da amostra sem pré-tratamento no cromatógrafo a gás. Isto torna-se mais crítico principalmente devido a baixa detectabililidade dos detectores cromatográficos e a indesejável contaminação da coluna por resíduos não-voláteis.

No presente trabalho utilizou-se o headspace dinâmico para a análise de poluentes em água. Esta técnica de introdução de amostra na coluna cromatográfica foi comparada com a injeção na coluna ("on-column"), principalmente quanto à sensibilidade da metodologia na análise de benzeno, tolueno e mistura dos xilenos (BTX) em amostras de água.

\section{PARTE EXPERIMENTAL}

\section{Material e Métodos}

Foram utilizadas as seguintes substâncias padrão: benzeno (Carlo Erba, Duque de Caxias - RJ/ Brasil), tolueno (Merck, Rio de Janeiro - RJ/ Brasil) e xileno (Merck, Rio de Janeiro RJ/ Brasil). Metanol, (grau pesticida, Carlo Erba, Duque de Caxias - RJ/ Brasil), foi usado como solvente.

\section{Injeção na Coluna (“On-column”)}

As soluções utilizadas foram preparadas em metanol. Para a determinação do tempo de retenção, os compostos foram injetados separadamente.

A partir de uma solução estoque de $1000 \mathrm{mg} / \mathrm{L}$ de BTX em metanol, foram preparadas as soluções mais diluídas para a obtenção da curva de calibração. Foi traçada uma curva de calibração para cada componente, em cada detector utilizado.

A análise cromatográfica foi realizada em um Cromatógrafo a Gás SRI-8610 (San Francisco/ USA), nas seguintes condições:

Coluna: LM-5 (5\% fenil 95\% dimetilpolissiloxano) de $15 \mathrm{~m}$ x $0,53 \mathrm{~mm}$ x $5 \mathrm{~mm}$ (São Carlos - SP/ Brasil).

Programação de temperatura:

- temperatura inicial: $40^{\circ} \mathrm{C}$

- rampa de aquecimento: $8^{\circ} \mathrm{C} / \mathrm{min}$.

- temperatura final: $120^{\circ} \mathrm{C}(5 \mathrm{~min})$

Foram utilizados os detectores por Fotoionização (PID) e por Ionização em Chama (FID), conectados em série. O volume injetado foi de $1 \mu \mathrm{L}$. 


\section{Headspace Dinâmico}

Utilizando-se o mesmo Cromatógrafo a Gás SRI-8610, conectou-se o sistema de Headspace Dinâmico, incluindo-se os "traps" de Tenax-GR e Carbopack B, conectados em série. A Figura 1 mostra o esquema de montagem do Headspace Dinâmico utilizado.

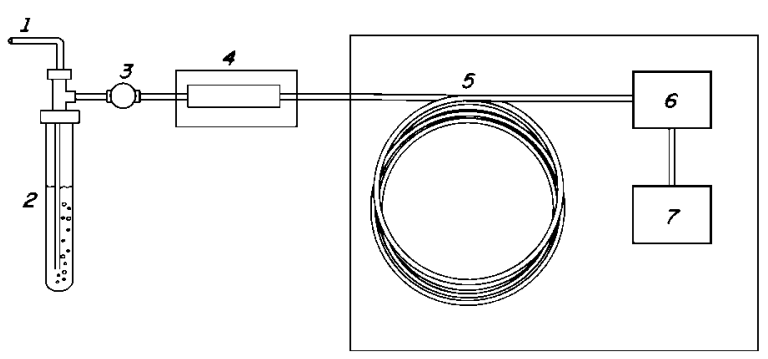

1. Entrada do gás de purga $\left(\mathrm{N}_{2}\right)$

5. Coluna cromatográfica

2. Tubo contendo amostra

6. Detector PID

3. Válvula

7. Detector FID

4. "Trap"

Figura 1. Esquema do sistema experimental de Headspace Dinâmico utilizado.

Todas as soluções de BTX foram preparadas com água purificada através de um Purificador de Água Milli-Q da Millipore. Foi preparada uma solução estoque de $1 \mathrm{mg} / \mathrm{L}$ e, a partir desta solução, foram preparadas soluções para a construção da curva de calibração.

Uma alíquota de $5 \mathrm{ml}$ de amostra foi introduzida no tubo de ensaio. A amostra foi purgada com $\mathrm{N}_{2}$ (nitrogênio ultrapuro grau ECD) com fluxo de $10 \mathrm{ml} / \mathrm{min}$, durante 4 minutos.

$\mathrm{O}$ extrato contendo os analitos voláteis da amostra foi transferido para os "traps" de dimensões $0,6 \mathrm{~cm}$ x $12 \mathrm{~cm}$.

A Programação de Eventos utilizada para o Headspace Dinâmico controlada por computador, foi:

0,10 min. - início da purga com nitrogênio

4,00 min. - fim da purga com nitrogênio

6,00 min. - início do aquecimento do "trap"

8,00 min. - injeção da amostra

12,00 min. - final do aquecimento do "trap"

Após a purga e aprisionamento, os analitos voláteis foram termicamente dessorvidos e automaticamente injetados. As condições cromatográficas foram:

Cromatógrafo a gás: SRI-8610

Coluna: LM-5 (5\% fenil 95\% dimetilpolissiloxano) de $22 \mathrm{~m} \mathrm{x}$ $0,53 \mathrm{~mm} \times 1,6 \mu \mathrm{m}$.

Programação de temperatura:

- temperatura inicial: $40^{\circ} \mathrm{C}(6 \mathrm{~min})$

- rampa de aquecimento: $10^{\circ} \mathrm{C} / \mathrm{min}$.

- temperatura final: $180^{\circ} \mathrm{C}$

No detector por fotoionização (PID) foi utilizada uma lâmpada de $10,2 \mathrm{eV}$.

Foram analisadas também algumas amostras de água potável da cidade de São Carlos, coletadas diretamente em pontos de consumo escolhidos aleatoriamente.

\section{RESULTADOS E DISCUSSÃO}

\section{Injeção na Coluna (“on-column”)}

As equações das retas obtidas a partir das curvas de calibração geradas por injeção na coluna (“on-column”) para benzeno, tolueno e xilenos, utilizando os detectores PID e FID, estão representadas na Tabela 1.

Tabela 1. Equações das retas obtidas a partir das curvas de calibração do Benzeno, Tolueno, Xileno obtidas por Injeção na coluna ("on-column"), utilizando os detectores PID e FID.

\begin{tabular}{lcc}
\hline & \multicolumn{2}{c}{ Equação da reta $(\mathrm{y}=\mathrm{a}+\mathrm{bx})$} \\
\hline & $\mathrm{a}$ & $\mathrm{b}$ \\
\hline Benzeno - FID & 5990,5 & 5,29 \\
Benzeno - PID & 0,91 & 8,16 \\
Tolueno - FID & 2453,9 & 4,91 \\
Tolueno - PID & $-1,49$ & 6,78 \\
Xileno - FID & 1222,4 & 6,11 \\
Xileno - PID & 1,44 & 4,56 \\
\hline
\end{tabular}

As análises feitas pelo método de injeção na coluna ("oncolumn"), ou seja introduzindo-se a amostra líquida diretamente na coluna cromatográfica, utilizando detector por ionização em chama (FID), foram possíveis somente até a concentração de $50 \mathrm{mg} / \mathrm{L}$ (50 ppm), devido ao fato do pico correspondente ao Benzeno estar eluindo na cauda do pico do solvente (metanol). Porém, no caso do detector PID, foi possível chegar a concentrações menores, ou seja, $0,5 \mathrm{mg} / \mathrm{L}(0,5 \mathrm{ppm})$ devido ao fato do detector ser mais seletivo, não sendo observado o pico do metanol, como mostra a Figura 2. a)

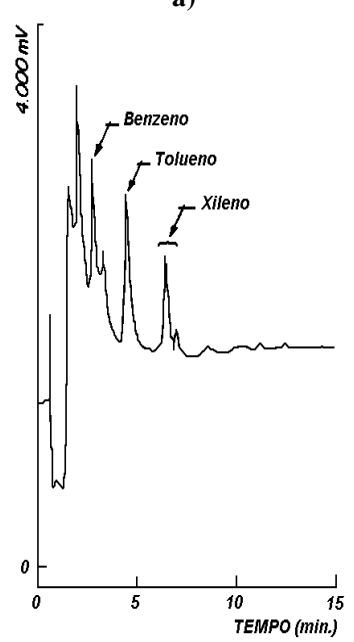

b)

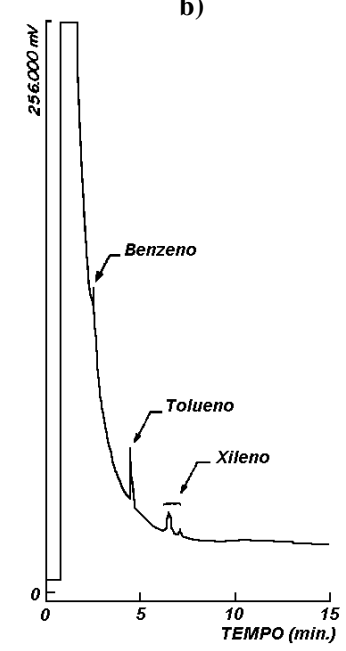

Figura 2. Cromatograma da mistura de BTX, obtido por Injeção na coluna ("on-column"), a) 0,5 $\mathrm{mg} / \mathrm{L}(0,5 \mathrm{ppm})$, utilizando detector PID; b) $50 \mathrm{mg} / \mathrm{L}(50 \mathrm{ppm})$, utilizando detector FID.

\section{Headspace Dinâmico}

Quando foi feito o teste com o Headspace Dinâmico, usando os "traps" de Tenax-GR e Carbopack B em série, observouse um "pico fantasma". Foi necessária, a retirada do trap de Tenax-GR para resolver este problema; a análise apenas com o trap de Carbopack B mostrou-se plenamente satisfatória.

As equações das retas obtidas a partir das curvas de calibração geradas por Headspace Dinâmico para benzeno, tolueno e xilenos, utilizando os detectores PID e FID, estão representadas na Tabela 2.

$\mathrm{Na}$ elaboração da curva de calibração utilizando o Headspace Dinâmico, verificou-se que os níveis mínimos de detecção de BTX foram de $1 \mu \mathrm{g} / \mathrm{L}$ de BTX, tanto no detector FID quanto no detector PID. Os cromatogramas obtidos na concentração de $1 \mu \mathrm{g} / \mathrm{L}$ estão representados na Figura 3. 
a)

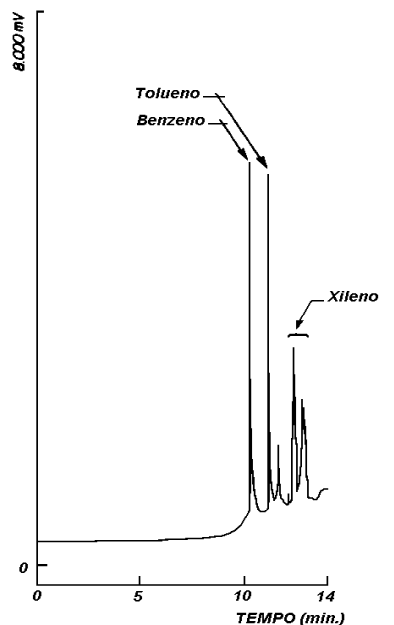

b)

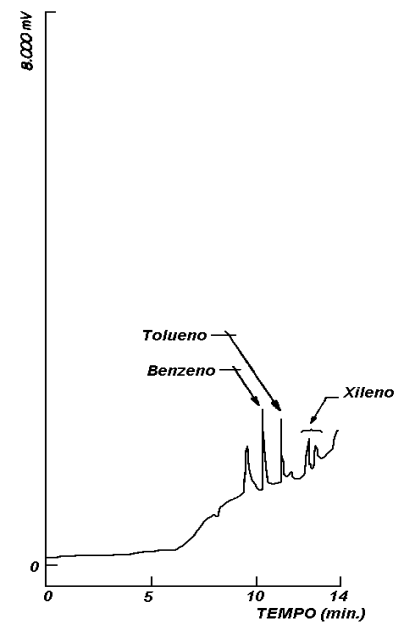

Figura 3. Cromatograma obtido na análise de $1 \mathrm{mg} / \mathrm{L}$ de BTX, obtido por Headspace Dinâmico, utilizando os detectores PID (a) e FID (b).
Tabela 2. Equações das retas obtidas a partir das curvas de calibração do Benzeno, Tolueno, Xileno utilizando o Headspace dinâmico, nos detectores PID e FID.

\begin{tabular}{ccc}
\hline & \multicolumn{2}{c}{ Equação da reta $(\mathrm{y}=\mathrm{a}+\mathrm{bx})$} \\
\hline & $\mathrm{a}$ & $\mathrm{b}$ \\
\hline Benzeno - FID & 3,82 & 4,96 \\
Benzeno - PID & 7,22 & 9,67 \\
Tolueno - FID & $-2,73$ & 10,58 \\
Tolueno - PID & 8,14 & 18,81 \\
Xileno - FID & 0,15 & 4,39 \\
Xileno - PID & $-9,32$ & 20,13 \\
\hline
\end{tabular}

Verificou-se também que até esses níveis obteve-se sinais limpos com poucos interferentes os quais, quando presentes, não eluem no mesmo tempo de retenção dos compostos analisados. A Figura 4, mostra os resultados da análise de amostra de água potável proveniente de poço artesiano e após ser submetida a tratamento pelo SAAE (Serviço Autônomo de Água e Esgoto).

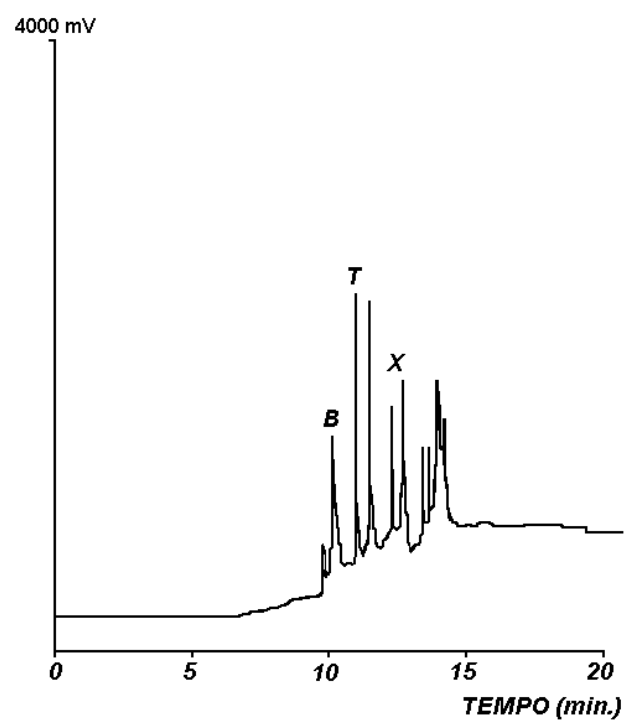

1a)

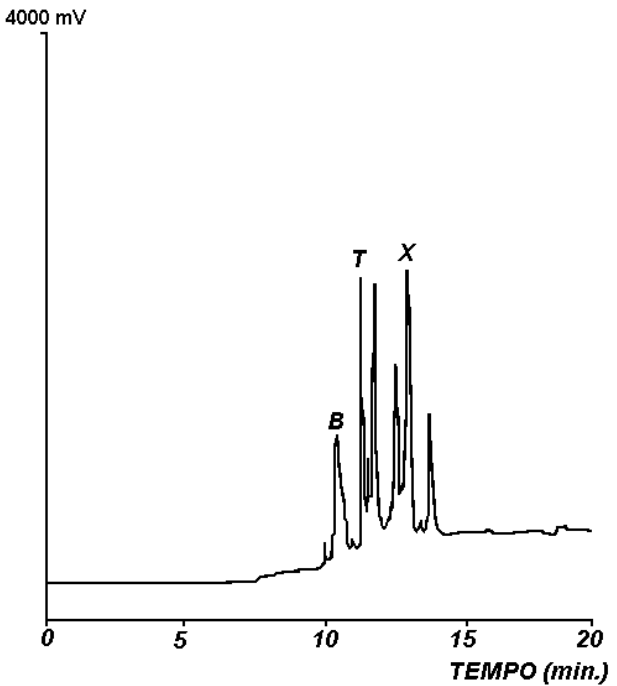

2a)

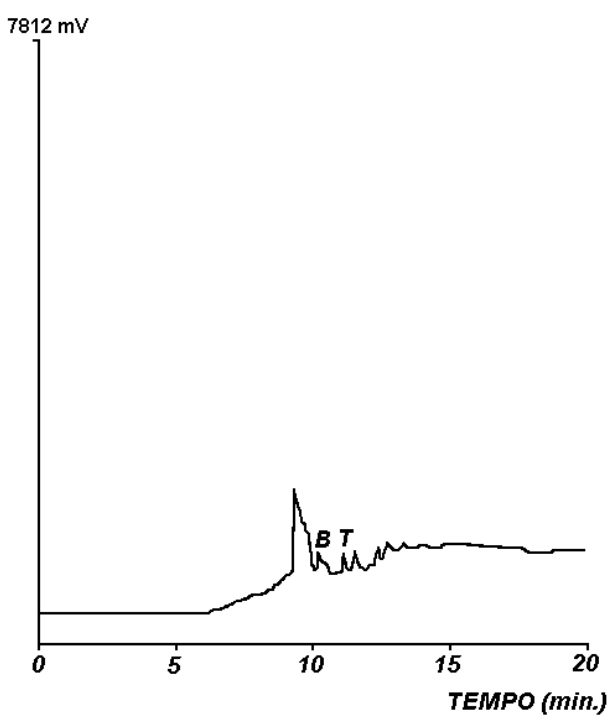

1b)

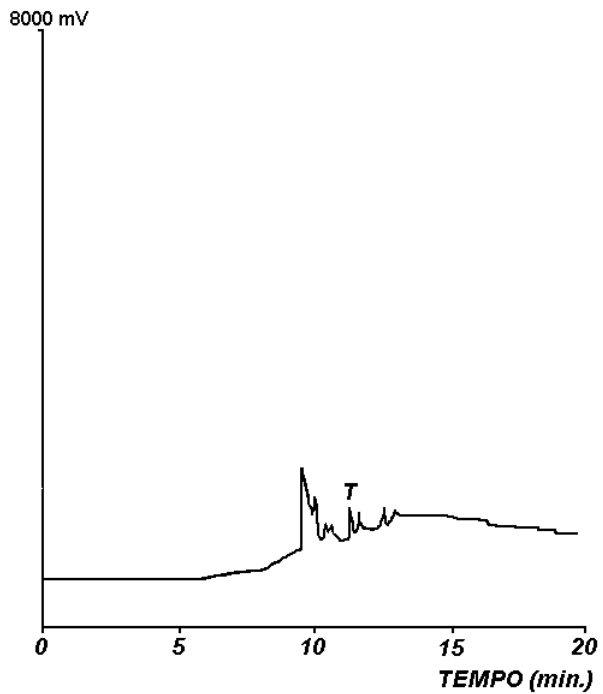

2b)

Figura 4. Cromatograma da Água potável de São Carlos proveniente de poço artesiano (1) e do tratamento feito pelo SAAE (2), obtido por Headspace Dinâmico, utilizando os detectores PID (a) e FID (b). 
No caso das amostras de água potável analisadas, os níveis de benzeno, tolueno e xileno determinados foram inferiores a $1 \mu \mathrm{g} / \mathrm{L}(1 \mathrm{ppb})$.

Verificou-se, também, que o FID não foi adequado para análise de BTX em nível de $\mu \mathrm{g} / \mathrm{L}$ (ppb).

\section{CONCLUSÕES}

Pode-se concluir que a análise por injeção na coluna ("oncolumn”) de BTX em água é possível, mas limitada pelos níveis mínimos de detecção de $50 \mathrm{mg} / \mathrm{L}(\mathrm{ppm})$ para o detector FID e $0,5 \mathrm{mg} / \mathrm{L}$ (ppm) para o detector PID.

Entretanto, com o método de "Headspace" Dinâmico ("purge-and-trap") foi possível analisar um volume maior de amostra $(5 \mathrm{~mL})$, chegando-se a respostas melhores uma vez que o "trap" age como um concentrador dos analitos. No caso do uso do detector FID, além da vantagem relativa ao "headspace" dinâmico, ainda existe o fato de que foi possível trabalhar em solução aquosa (para concentrações baixas de BTX é possível solubilizá-los em água) eliminando, assim, o problema do pico interferente (Metanol) quando analisamos as soluções de padrões analíticos.

Analisando-se os resultados obtidos até o momento, pode-se concluir que o "headspace" dinâmico é capaz de detectar compostos em níveis de ng/L (ppt), sendo que esta detectabilidade poderia ser melhorada através do aquecimento da amostra. Isto faz com que esta técnica seja uma das mais indicadas para a análise de compostos voláteis em água potável, onde espera-se uma quantidade pequena de contaminantes, em baixas concentrações.

\section{AGRADECIMENTOS}

Os autores agradecem a FAPESP (Proc. 1996/9269-5) e CNPq (Proc. 523.300/95-6) pelo suporte financeiro ao nosso laboratório.

\section{REFERÊNCIAS}

1. Monticeli, J. J.; Martins, J. P. S. A Luta pela água - Nas Bacias dos Rios Piracicaba e Capivari. $1^{\text {a }}$ ed., Capivari, EME, 1993.

2. Tap Water Consumption in Canada; Department of National Health and Welfare, Ottawa, 1981; Report $n^{\circ}$ 82-EHD-80.

3. Pelon, W.; Whitman, B.F.; Beaslev, T. W.; Environ. Sci. Technol. 1977, 11, 619.

4. Oliver, B. G.; Charlton, M. N.; Environ. Sci. Technol. 1984, 18, 903.

5. Lanças, F. M. Cromatografia em Fase Gasosa, São Carlos SP, Acta, 1993.

6. Soleta, D. D.; Am. Lab. 1989, 21, 21. 